CAHIERS DE

NARRATOLOGIE

\section{Cahiers de Narratologie}

Analyse et théorie narratives

32 | 2017

Récit et argumentation, interactions, lieux et dispositifs sociaux

\title{
U. Eco et le storytelling conspirationniste
}

Arguments et contre-arguments

\section{Alessandro Leiduan}

\section{(2) OpenEdition}

\section{Journals}

Electronic version

URL: http://journals.openedition.org/narratologie/7800

DOI: $10.4000 /$ narratologie. 7800

ISSN: 1765-307X

Publisher

LIRCES

Electronic reference

Alessandro Leiduan, « U. Eco et le storytelling conspirationniste », Cahiers de Narratologie [Online],

32 | 2017, Online since 21 December 2017, connection on 19 April 2019. URL : http://

journals.openedition.org/narratologie/7800; DOI : 10.4000/narratologie.7800

This text was automatically generated on 19 April 2019

Article L.111-1 du Code de la propriété intellectuelle. 


\title{
U. Eco et le storytelling conspirationniste
}

\author{
Arguments et contre-arguments
}

\section{Alessandro Leiduan}

1 Pourquoi la société croit-elle aux théories du complot? Pourquoi est-elle de plus en plus vulnérable à des réécritures de l'histoire officielle qui n'ont aucun fondement empirique ? Pourquoi permet-elle à des croyances infondées de se glisser clandestinement parmi les croyances évidentes et consensuelles qui devraient délimiter le sens social de la réalité ? Je voudrais essayer de répondre à ces questions en interrogeant l'œuvre d'un auteur qui s'est intéressé à ce phénomène bien des années avant que le conspirationnisme ne commence à s'imposer à l'attention de la critique. U. Eco s'est, en effet, penché sur ce thème dès son deuxième roman, Le Pendule de Foucault, écrit en 1988.

2 Il semblerait que, pour interpréter l'œuvre d'un écrivain, l'interprète devrait s'éclipser complètement de manière à restituer le plus fidèlement possible la pensée de cet auteur sans y incorporer des développements qui lui sont étrangers. Mais, à bien y réfléchir, ce parti pris condamnerait l'interprète à n'accoucher que d'une froide reformulation tautologique de la pensée de l'auteur interprété sans pouvoir apporter le moindre éclaircissement aux points obscurs ou aux apories que toute pensée porte forcément en elle. J'emprunterai donc le chemin inverse et reconstituerai la pensée d'U. Eco sur le conspirationnisme en essayant de dialoguer avec elle, en marquant mes différences et en problématisant les divergences entre nos points de vue respectifs, de manière à permettre au lecteur de se positionner librement par rapport aux thèses et aux arguments mis en jeu.

\section{Définition du problème}

3 L'expression « théories du complot » recouvre l'ensemble des discours qui font planer sur le cours de l'Histoire l'ombre du complot. Selon ces théories, l'Histoire humaine comporterait une "partie visible» et une «partie invisible»: la partie visible coïnciderait avec tous les faits qui sont mentionnés dans les manuels d'histoire, la partie 
invisible correspondrait aux différents complots que trameraient des personnages invisibles dans le but d'asseoir leur pouvoir, tout en donnant l'illusion au reste de l'humanité de vivre dans un monde qui n'obéit qu'à la volonté d'un peuple libre, autonome et, surtout, capable de s'autodéterminer. Selon les théories du complot, il n'y aurait, en revanche, qu'une seule et unique protagoniste de l'Histoire : la société secrète. Les figures que les hommes créditent du statut de "personnages historiques " ne seraient, en réalité, que des marionnettes exécutant un plan d'action qui a été conçu, planifié et ficelé par la société secrète. Celle-ci dissimulerait son existence derrière un respect formel des institutions démocratiques. Les théories du complot révéleraient ainsi le côté obscur des démocraties modernes, c'est-à-dire les vices secrets qui polluent les valeurs autour desquelles devrait tourner la vie politique. Elles montreraient que la vraie fonction des valeurs en question (la justice, l'égalité, la liberté) n'est peut-être pas tant celle de forcer la société à dépasser, au nom de l'intérêt général, les égoïsmes personnels, corporatifs ou de classe, mais plutôt celle de dissimuler l'égoïsme le plus sordide, en lui permettant de se déployer en toute impunité. Les théories du complot révéleraient donc le cancer qui se développe dans le corps des sociétés démocratiques - un cancer qui menacerait, à terme, leur existence (ou, du moins, leur authenticité).

Il n'est pas étonnant, dès lors, que ces théories soient si sévèrement critiquées. L'hostilité à l'égard des théories du complot tient au fait qu'elles mettent à mal la crédibilité de l'histoire officielle, l'empêchant de remplir sa fonction idéologique. L'Histoire, en effet, n'est pas seulement un savoir sur les époques passées de l'humanité. L'Histoire remplit aussi une fonction de légitimation idéologique de l'ordre existant. Toute société existante cherche dans son passé un reflet d'elle-même, une manifestation embryonnaire des valeurs en lesquelles elle croit. Les faits mentionnés dans les livres d'Histoire sont autant d'exemplifications des valeurs autour desquelles les sociétés existantes ont construit leur mode de vie et, plus particulièrement, leur modèle d'organisation politique. Les théories du complot sont critiquées parce qu'elles empêchent la société de se complaire dans l'image que les livres d'Histoire lui renvoient. Malmenée par les réécritures conspirationnistes, l'Histoire ne rend plus compte, en effet, de l'épopée d'une société qui, après s'être affranchie de l'ignorance, de la superstition et de l'exploitation, a su se doter d'un modèle politique démocratique, faisant de la "chose publique » l'affaire de tous et non pas seulement d'un monarque absolu, intronisé par un Dieu tout-puissant. L'Histoire célèbre plutôt le triomphe de la tromperie, de la machination et de la dissimulation, ingrédients typiques de tout complot. Si un nombre croissant de personnes, au sein de notre société, s'accommode désormais de ce genre de scénarios, c'est que l'épopée qui aurait présidé au tournant démocratique de l'histoire humaine laisse sceptique un nombre de plus en plus important de personnes. Doit-on s'en étonner? Pas vraiment. Les sociétés qui se réclament aujourd'hui d'un héritage démocratique sont le plus souvent régies par une logique économique qui ne fait plus grand cas des règles de la démocratie, ses seuls critères de réussite étant l'augmentation de la richesse, l'intensification des échanges et l'addiction des masses à la consommation. Certes, les sociétés démocratiques à régime capitaliste ne sont pas complètement dépourvues d'une éthique, d'une politique ou d'une religion. Mais, en cas de conflit entre les valeurs éthiques, politiques ou religieuses, d'un côté, et les intérêts économiques, de l'autre, les sacrosaints principes de l'économie tendent à l'emporter (Michéa 2007). L'ultra-capitalisme actuel demande à l'humanité de sacrifier ses propres convictions éthiques, politiques et religieuses sur l'autel de la rationalité économique. La société est ainsi éduquée à s'affranchir de cet 
ensemble de valeurs spirituelles autour duquel gravitait la vie symbolique des sociétés du passé. Max Weber caractérisait cette décomposition de la vie symbolique attestée dans toutes les sociétés occidentales modernes en parlant de « désenchantement du monde » ( Enzauberung der Welt). Limité, en son temps, aux valeurs religieuses, le « désenchantement » en question concerne désormais également d'autres sphères de la vie symbolique : l'esthétique, l'éthique et la politique. Sans être devenues complétement " obsolètes ", les valeurs autour desquelles gravitait la vie communautaire des sociétés du passé sont aujourd'hui de simples "prothèses idéologiques d'une dimension de l'existence complètement factice et vidée de toute souveraineté » (Preve 2007). Comme la conception sociale de la réalité était imprégnée de ces valeurs, le déclin de celles-ci a eu pour conséquence de fragiliser la conception sociale de la réalité, qui s'est ainsi laissée coloniser par des croyances infondées de nature conspirationniste.

Le point de vue d'Eco sur la question est tout à fait différent. Selon lui, c'est dans l'univers de la fiction que se trouveraient les archétypes de la théorie du complot, des fausses nouvelles et des autres croyances mensongères qui polluent la vision sociale de la réalité. Ces croyances ne seraient donc que des excroissances fictionnelles de l'imaginaire social. La littérature fictionnelle nous parle d'ogres qui mangent des enfants, de sorcières qui ont des rapports avec le diable, de fanatiques qui conspirent contre le reste de l'humanité... Quand nous lisons toutes ces choses, nous sommes persuadés de ne pas y croire. Mais il suffit, observe Eco, que l'on imagine un seul instant que notre pire ennemi pourrait avoir accompli l'une de ces monstruosités pour être tentés de croire vraiment qu'il existe au monde des personnes assez perverses pour commettre des choses aussi obscènes. Les scénarios les plus invraisemblables des romans gothiques, des romans feuilletons, des films d'épouvante se réalisent inopinément dans le monde réel. Enivrés par le plaisir qui nous envahit lorsque nous imaginons les personnes que nous haïssons viscéralement dans l'acte de dévorer des enfants ou de copuler avec le diable ou de conspirer contre le reste de l'humanité, nous finissons par croire vraiment qu'ils se comportent ainsi. Il a été donc possible, selon Eco, d'accuser de ces monstruosités les Templiers, les sorcières, les Juifs, les francs-maçons, etc.

les gens dévorent les aventures de terre ou de mer ou les histoires criminelles par simple plaisir, puis oublient facilement ce qu'ils ont appris et, quand on leur fait prendre pour argent comptant ce qu'ils ont lu dans un roman, ils ne s'avisent que vaguement qu'ils en avaient déjà entendu parler, et ils ont confirmation de leurs croyances (Le cimetière de Prague : 372 )

6 Tous les lecteurs des romans d'Eco savent que son œuvre narrative, même si elle semble multiforme et variée, ne fait que décliner à l'infini une seule et même histoire : l'odyssée de personnages qui perdent le sens de la réalité et finissent par croire aux histoires imaginaires qu'ils avaient eux-mêmes inventées. Un menteur comme Baudolino finit par croire aux reconstructions étoffées de mensonges de sa propre vie et de l'histoire de son temps. Un personnage du Pendule de Foucault prétend être la réincarnation du légendaire Comte de Saint-Germain. Roberto de la Grive se persuade de pouvoir réécrire, avec la seule force de l'imaginaire, le cours entier de l'Histoire sacrée (L'̂̂le du jour d'avant ). Simone Simonini croit à l'existence d'un complot juif contre le reste du monde, tout en ayant lui-même inventé de toutes pièces cette histoire (Le cimetière de Prague). C'est en mettant en scène l'égarement mental de ses personnages qu'Eco espère pouvoir mettre à l'abri ses propres lecteurs du risque de s'engouffrer dans les mêmes erreurs.

7 Reste à voir - et ce sera l'enjeu des lignes suivantes - si Eco n'est pas lui-même atteint de ce donquichottisme qu'il impute à ses personnages. Reste à voir si l'implication de la 
fiction dans la propagation des théories du complot est vraiment crédible. Reste surtout à voir si le prix qu'Eco fait payer à ses romans pour les rendre socialement acceptables, c'est-à-dire pour éviter qu'ils deviennent le lieu d'incubation de nouvelles théories du complot, n'est pas trop élevé par rapport à celui qu'une œuvre de fiction peut payer sans cesser pour autant d'exister en tant qu'œuvre de fiction. $\mathrm{Si}$, en effet, pour rendre socialement acceptable un roman, on l'empêche de remplir la fonction à laquelle les romans sont tributaires de leur utilité culturelle, la réhabilitation sociale prétendue de ce genre littéraire ne se transforme-t-elle pas peut-être en une condamnation à mort?

\section{Les arguments du réquisitoire d'Eco contre le conspirationnisme}

Pourquoi, selon Eco, les gens succombent-ils à des croyances fictionnelles?

Eco fournit au moins deux réponses:

(i) Les gens ne croient pas aux choses vraies, mais aux choses vraisemblables.

Il n'est pas nécessaire qu'une croyance soit vraie pour pouvoir être crue. Il suffit qu'elle soit vraisemblable. Nous avons tendance à tenir pour crédible toute affirmation qui confirme notre vision du monde et nous ne nous embarrassons pas de savoir s'il y a des preuves exemptes de doute et d'incertitude qui valident l'affirmation en question. «... quand un espion vend quelque chose d'inédit - affirme un des personnages d'Eco - il ne doit rien faire d'autre que raconter quelque chose qu'on pourrait trouver sur tout éventaire de livres d'occasion » (Le cimetière de Prague : 141). Il n'y a rien, selon Eco, qui ne puisse devenir objet de croyance, y compris les choses les plus invraisemblables. Les gens ne veulent apprendre que ce qui peut flatter leurs préjugés, surtout s'il s'agit de préjugés qui n'ont aucune légitimation empirique. «Il m'est toujours arrivé - affirme Baudolino qu'à peine je disais j'ai vu ça, ou bien j'ai trouvé cette lettre qui dit comme ça (avec même la possibilité que je l'aie écrite moi), les autres donnaient l'impression qu'ils n'attendaient que cela » (Baudolino: 46). Et ce même personnage ajoute un autre exemple: «S'il me passait par la tête de dire que j'avais vu une sirène dans la mer (...), tous y croyaient et me disaient épatant épatant... » (53). En tant que creuset d'histoires imaginaires, la fiction est donc, d'après Eco, le lieu d'incubation des croyances sociales les plus bizarres (parmi lesquelles, bien évidemment, les théories du complot).

11 (ii) La fiction ne peut être falsifiée.

Les genres fictionnels sont par définition des genres imaginaires. Ils stimulent l'imaginaire social au moyen d'histoires inventées de toutes pièces. Il s'ensuit qu'il n'existe aucun document écrit, aucun témoignage oral ou, plus en général, aucune donnée empirique susceptible de légitimer l'authenticité des choses racontées dans un roman. Si nous croyons donc aux histoires divulguées par les romans ou à l'existence d'un personnage créé par l'imagination d'un romancier, personne n'aura jamais la possibilité de délégitimer nos croyances, faute de pouvoir réfuter les documents empiriques sur lesquels devraient s'appuyer les croyances en question. Selon Eco, les croyances sociales les plus difficiles à extirper sont, paradoxalement, celles qui ne s'appuient sur aucun document empirique. S'il existe des documents empiriques à l'appui de la diffusion d'une nouvelle, il y a toujours la possibilité de délégitimer cette nouvelle en dénonçant le caractère non-authentique du document en question. Ainsi, Lorenzo Valla, humaniste italien du XVe siècle, a pu démontrer que la Donatio Constantini, par laquelle les papes 
prétendaient que l'empereur Constantin leur avait délégué le pouvoir, était un faux document, fabriqué de toutes pièces quelques siècles après la mort de l'empereur. Or, selon Eco, les fausses nouvelles les plus redoutables sont celles qui s'appuient sur des racontars, des témoignages indéterminés, mais surtout sur des œuvres de fiction. "Jamais, jamais au grand jamais il ne faut travailler sur des documents authentiques, ou authentiques à moitié! S'ils existent quelque part, quelqu'un pourra toujours aller les chercher et démontrer que quelque chose a été reporté de façon inexacte... » (Le cimetière de Prague : 261).

\section{La réforme du « pacte fictionnel » ou le remède d'Eco contre le storytelling conspirationniste}

Persuadé que la perception sociale de la «réalité» est contaminée par des représentations de matrice romanesque, Eco a voulu éviter que ses propres romans ne deviennent eux aussi des foyers involontaires de croyances conspirationnistes. A cet effet, il a adopté une série de mesures visant à empêcher toute interaction entre les significations véhiculées par ses romans et l'ensemble des paradigmes à la lumière desquels la société déchiffre toute expérience empirique (la « réalité » proprement dite). C'est la fiction même, chez lui, qui s'auto-dénonce comme fausse, mensongère, en un mot, « incroyable». En tant que romancier, Eco a signé des romans qui s'offrent au lecteur sous des apparences qui n'autorisent aucun acte de croyance. Au lieu d'entretenir son public dans l'illusion que l'histoire racontée pourrait être vraie, il s'est appliqué à démasquer le caractère absolument artificiel, invraisemblable et faux de ce qu'il a raconté. Son but est d'enfermer hermétiquement les significations véhiculées par ses romans dans un cadre étanche d'où il n'est plus possible de les faire sortir afin de préserver l'immutabilité de la perception de la réalité la plus diffuse et conventionnelle.

Les stratégies qui permettent à Eco de porter le coup de grâce à la crédibilité de ses histoires sont les suivantes:

a) Sur le plan de l'énonciation, Eco confie le récit à deux narrateurs différents : l'un reçoit (à l'écrit ou à l'oral) le témoignage de l'autre et, après l'avoir émondé de certaines parties, en entreprend le récit dans la version que découvre le lecteur du roman. Une même histoire est donc prise en charge par deux voix différentes : celle d'un narrateur-témoin qui raconte à la première personne une aventure qu'il a directement vécue, et celle d'un narrateur-éditeur qui reformule avec ses propres mots l'aventure qui lui a été racontée. Or, le narrateur-témoin est généralement présenté comme un être crédule, prêt à gober toute sorte d'histoire fausse, y compris celles qu'il a lui-même inventées. Voici ce que Baudolino dit de lui-même : "Quand tu dis une chose que tu as imaginée, et que les autres te disent qu'il en est vraiment ainsi, tu finis par y croire toi-même » (Baudolino : 46). Et voici ce que dit de lui-même Iacopo Belbo, narrateur-témoin du Pendule de Foucault : «J'étais en train de me demander qui nous sommes, nous. Nous qui croyons Hamlet plus vrai que notre concierge. Ai-je le droit de les juger, eux, moi qui rôde à la recherche de Madame Bovary pour lui faire une scène? " (Le Pendule de Foucault : 349). Amnésique, le narrateur-témoin du Cimetière de Prague cherche à retrouver la mémoire en rédigeant sa biographie, mais l'abus de drogue prive sa parole de toute crédibilité : «L'absinthe est en train d'accomplir son œuvre, et si j'haleinais sur une bougie je déclencherais une grande flamme au lumignon» (220). A la fin, il avoue : «La vérité est que peut-être je suis fou» (300). Et 
c'est, à peu près, dans les mêmes termes que l'on qualifie le personnage de Braggadocio dans le roman Numéro Zéro (127 et 215).

Quant au narrateur-éditeur, sa fonction est de mettre constamment en doute la parole du narrateur-témoin en destituant son récit de toute crédibilité : " Je suis plein de doutes ", écrit, par exemple, le narrateur du Nom de la rose, dans la préface du roman. «Je ne sais vraiment pas pourquoi je me suis décidé à prendre mon courage à deux mains pour présenter comme s'il était authentique le manuscrit d'Adso de Melk» (Le Nom de la rose : 15). La même chose est observable dans les autres romans d'U. Eco. Voici ce que dit le narrateur-éditeur de Numéro zéro du personnage qui remplit, dans ce roman, la fonction de narrateur-témoin : «Je ne comprenais pas si Braggadocio était un génial conteur de feuilleton , qui dosait son roman à épisodes avec ce qu'il fallait de suspense à chaque "à suivre", ou si vraiment il reconstituait sa trame morceau par morceau" (Numéro zéro: 161). Le narrateur-éditeur du Cimetière de Prague exprime ouvertement sa perplexité sur l'authenticité de l'histoire qu'il raconte au lecteur: «Vous admettrez que le Narrateur aussi soit perplexe. C'est que l'Abbé Dalla Piccola semble se réveiller seulement quand Simonini a besoin d'une voix de la conscience qui accuse ses distractions et le rappelle à la réalité des faits (...). A le dire sans ambages, si ces pages ne rapportaient pas des choses absolument vraies, il paraîtrait que ce serait l'art du Narrateur qui a disposé ces alternances d'euphorie amnésique et de dysphorique mémoire " (Le cimetière de Prague: 223). Le narrateur-éditeur du roman Baudolino en vient même à revendiquer ouvertement le statut de «menteur»: «Ne te crois pas l'unique auteur d'histoires en ce monde - s'exclame un personnage à la fin du roman - Tôt ou tard, quelqu'un, plus menteur que Baudolino, la racontera.» (Baudolino: 665). Ce «menteur » plus menteur que Baudolino n'est autre que le narrateur anonyme qui raconte l'histoire de ce personnage. Quant au narrateur de l'île du jour d'avant, il reconnaît avoir fait semblant de raconter la vérité : aveu incompatible avec les règles du jeu fictionnel: «où, certes, on fait semblant de raconter des choses vraies, mais on ne doit pas dire sérieusement qu'on fait semblant » ( L'île du jour d'avant : 2051).

b) Sur le plan de l'énoncé, Eco bat en brèche tous ou presque les systèmes de prévisions qui président à la compréhension des comportements humains représentés dans un roman. En règle générale, l'agir d'un personnage ne nous devient compréhensible que s'il se déploie selon des formes qui cadrent avec l'horizon prévisionnel à partir duquel nous déchiffrons l'agir de nos semblables. Le comportement d'oiseaux ou de poissons qui pensent ou parlent comme des hommes ou, à l'inverse, d'êtres humains qui marchent sur les nuages ou vivent dans les profondeurs sous-marines ou, plus en général, d'êtres vivants auxquels adviennent des choses absurdes que notre vision de la réalité juge « impossibles" - tous ces comportements ne sont interprétables qu'à la faveur d'une transposition allégorique de leurs significations littérales. Mais le recours à ce type d'interprétation est la conséquence d'une invraisemblance constitutive de l'histoire racontée. Or, Eco semble justement mettre un zèle particulier dans chacun de ses romans pour raconter des histoires qui se déploient sous le signe de l'invraisemblance la plus totale. Simonini, simple apprenti chez un notaire de Turin, entre au service de la police secrète du Règne de Sardaigne d'abord, et de l'Etat français, ensuite. En tant qu'espion, il entre en contact avec d'importantes figures de son époque: Garibaldi, Dumas, Ippolito Nievo, Maurice Joly, Freud. Il participe à l'expédition des Mille, prémisse de l'unification politique de l'Italie, à la Commune de Paris et à d'autres faits majeurs de l'histoire du XIXe siècle. Mais, surtout, il consacre sa vie à persécuter les Juifs et se distingue, dans ce 
domaine, par la réalisation d'un «exploit » effroyable, mais décisif: la rédaction de l'évangile de l'antisémitisme moderne, Les protocoles des Sages de Sion, qui servira ensuite de pièce maîtresse aux persécutions contre les Juifs, décidées par Hitler. Non que les faits racontés dans ce roman soient vraiment «impossibles", mais ils sont hautement « invraisemblables », c'est-à-dire qu'ils n'arrivent que très rarement ou, en tout cas, pas le plus souvent. Or, comme l'a souligné Aristote, le vraisemblable est «ce qui arrive le plus souvent" (Rhétorique, I (A), 2, 1357 a) et, à tout prendre, "l'impossible qui est vraisemblable est à préférer au possible qui est incroyable " (Poétique, 24, $1460 \mathrm{a}^{2}$ ). On constate chez d'autres héros des romans d'Eco la même tendance à réaliser des entreprises mémorables, peu compatibles avec leur statut social. Issu d'une modeste famille de la noblesse d'épée piémontaise, Roberto de la Grive, côtoie, dans L'Ile du jour d'avant, les grands noms de l'Histoire de son temps et reçoit de Mazarin en personne l'ordre d'embarquer sur un navire, l'Amarillis, pour espionner les Anglais. Simple paysan piémontais, Baudolino devient, dans le roman homonyme, le fils adoptif de l'empereur Frédéric Barberousse ainsi que son principal conseiller : il parvient ainsi à tirer les ficelles de toute l'histoire de son temps et à consacrer la gloire de son protecteur grâce à des ruses tout aussi spectaculaires qu'improbables (la création de fausses reliques, la canonisation de Charlemagne, etc.). Je le répète : les intrigues des romans d'Eco ne sont pas «impossibles", mais leur "vraisemblance » parait extrêmement douteuse: les destins de Simonini, de Roberto de la Grive, de Baudolino, même s'ils venaient à se réaliser dans la vie réelle, ils ne représenteraient qu'une exception à la règle qui préside au destin réel des hommes de notre espèce (dont on sait - ou l'on devrait savoir - qu'ils ne parviennent que très rarement à s'affranchir du déterminisme inscrit dans leur condition sociale et à façonner leur vie au gré de leur libre volonté toute-puissante). Aristote l'a d'ailleurs souligné à plusieurs reprises : la fiction s'est constituée, dans notre tradition culturelle, comme la mimésis d'une action vraisemblable, c'est-à-dire d'une action qui, par rapport à toutes celles qui rendent compte de la diversité des formes à travers lesquelles peut se déployer l'agir humain, correspond à l'action la plus probable qui soit. La liste des invraisemblances attestées dans les romans d'Eco serait encore longue, mais le constat ne changerait pas. Toutes les aventures de ses personnages offensent la manière dont une personne normale tend à se représenter le destin d'un être humain normal dans un monde normal. Mais ces invraisemblances, dans les intentions d'Eco, ne sont pas un défaut : elles mettent à l'abri ses fictions du soupçon de pouvoir fonctionner comme source de corruption de la perception sociale de la réalité. Eco en effet ne conçoit pas la fiction en termes de ressemblance, mais en termes de dissemblance avec le monde réel. Le lecteur d'un roman peut «faire semblant » de croire à un énoncé fictionnel uniquement lorsqu'il « découvre des divergences (discrepanze) entre le monde de son expérience et celui de l'énoncé luimême» (Eco 1979: 76). Eco imite sommairement l'ensemble des caractères conventionnels qui définissent la perception sociale de la réalité (les paradigmes descriptifs de la réalité culturelle), mais il le fait juste pour rendre compréhensible l'histoire racontée et ne manque jamais de signifier à ses lecteurs que l'histoire qu'ils lisent n'est pas réelle, mais imaginaire (et qu'elle comporte donc des faits qui ne se produisent pas dans la réalité). Le système prévisionnel qui préside à la compréhension de l'agir humain reste le terme de comparaison des histoires imaginaires inventées par Eco, mais uniquement dans le but de rendre évident qu'il existe un écart insurmontable entre la manière d'agir de ses personnages et la manière dont nous déchiffrons l'agir de nos semblables dans le monde « réel » où nous vivons. 
17 La réhabilitation de la fiction passe donc, chez Eco, par une révision du «pacte fictionnel» qui devrait déterminer l'attitude mentale de la société à l'égard des significations que les genres fictionnels véhiculent. Au lieu de permettre à la société de reconnaître dans ces significations un « miroir » de la réalité, Eco transforme ses romans dans une vitrine des comportements les plus invraisemblables que l'on puisse imaginer. La perception sociale de la réalité est, certes, mise à l'abri d'intempestives contaminations fictionnelles, mais l'inconvénient est que la fiction cesse d'être un lieu de problématisation de l'expérience vécue. Or, c'est en problématisant les catégories éthiques, politiques, religieuses et esthétiques qui président aux jugements que nous portons sur le monde et sur les choses qui s'y déroulent que la fiction a acquis - comme on le verra - ses lettres de noblesse dans notre tradition culturelle. Contrairement à ce que pense Eco, je considère que la fiction a contribué, non pas à corrompre, mais à affiner la perception sociale de la réalité.

\section{Plaidoyer pour la fiction : contre-arguments à la thèse d'Eco}

Récapitulons: selon Eco, la société actuelle serait atteinte d'un état d'aliénation imaginaire induit par la fiction. "A imaginer d'autres mondes, pourrait-il dire avec l'un de ses personnages, on finit par changer aussi celui-ci » (Baudolino : 104) La vulnérabilité de la société aux croyances conspirationnistes tiendrait notamment aux facteurs suivants : (i) l'indexation de la plupart des croyances sociales sur le vraisemblable et non pas sur la vérité empirique et (ii) l'impossibilité de délégitimer par des procédures de falsification empirique toute croyance empruntée à l'univers de la fiction. Il s'ensuit que, d'après lui, (iii) la fiction n'est légitime que si elle devient la vitrine des histoires les plus invraisemblables, de manière à court-circuiter les réflexes culturels qui, jadis, nous inclinaient à chercher dans les œuvres fictionnelles un reflet analogique de notre expérience du monde.

Qu'est-ce qu'on peut objecter aux thèses d'Eco?

(i) « La caractéristique principale des gens - peut-on lire dans le Cimetière de Prague - c'est qu'ils sont prêts à tout croire » (373). Que les gens croient les choses les plus bizarres, cela n'est pas impossible. Mais qu'ils s'accommodent des croyances les plus insensées sans exiger, avant d'y croire, qu'elles soient un tant soit peu crédibles, c'est plutôt difficile à accepter. Or, les fictions dans lesquels puiseraient leurs racines, selon Eco, les lubies conspirationnistes de notre société sont toutes des fictions hautement invraisemblables: romans feuilletons $\left(\mathrm{Sue}^{3}\right)$, romans de cape et d'épée $\left(\right.$ Dumas $\left.^{4}\right)$, contes merveilleux ou légendaires (Rabbi Löw $w^{5}$ ), etc. Sa thèse pourrait être tenue pour acceptable uniquement s'il n'existait aucun autre genre fictionnel que les romans feuilletons, les romans de cape et d'épée, les contes merveilleux ou légendaires. La fiction devrait, en somme, coïncider avec la paralittérature ${ }^{6}$. Mais il est évident qu'il existe aussi d'autres genres de fiction : la vulnérabilité imaginaire qui caractérise les lecteurs des fictions dont parle Eco ne peut donc pas être étendue à l'ensemble des lecteurs de fictions. Et l'on ne peut pas affirmer non plus que c'est l'indexation des croyances sociales sur les choses vraisemblables qui nous rend vulnérables aux récits conspirationnistes mis en circulation par la fiction: il est notoire, en effet, que les genres paralittéraires sont la vitrine de tout ce qu'il y a de plus invraisemblable dans le domaine de la fiction. 
21 (ii) Quant à l'impossibilité de délégitimer les croyances divulguées par une fiction, elle ne doit pas nous faire oublier que l'absence de documents empiriques joue aussi en sens inverse: elle met également en échec les tentatives de légitimation des croyances fictionnelles. Si l'absence de documents empiriques est préjudiciable à ceux qui voudraient réfuter une croyance divulguée par un roman, il est vrai aussi que l'absence de ces documents porte également préjudice à ceux qui voudraient légitimer cette croyance. Il n'est pas concevable que dans un monde imbu de scientisme qui reconnaît dans le savoir scientifique le parangon insurpassable de toute forme de savoir, l'absence de preuves empiriques soit propice à l'enracinement de croyances fictionnelles.

(iii) Voici enfin comment je critiquerais la conclusion qu'Eco tirait des prémisses (erronées) de son raisonnement sur les rapports entre fiction et théories du complot. Point n'est besoin pour réhabiliter socialement la fiction de priver les représentations qu'elle met en circulation de toute vraisemblance: les représentations en question, si vraisemblables soient-elles, n'ont pas vocation à prendre la place de la "réalité ». Le " semblant » de réalité qu'on peut y déceler est fonctionnel à la constitution de la fiction en tant que telle ${ }^{7}$. Celle-ci ne peut exister que si l'on fait semblant de croire à la réalité des représentations qu'elle met en scène, tout en étant conscient qu'elles sont irréelles. Lorsqu'un romancier, par exemple, nous raconte une histoire, nous savons parfaitement que cette histoire est irréelle, mais nous adoptons vis-à-vis d'elle la même attitude mentale qui serait la nôtre si elle était "réelle » : nous «faisons semblant de croire » à l'histoire en question, nous essayons de la " vivre " par l'intermédiaire des personnages, nous tâchons d'imaginer ce que nous aurions ressenti si nous l'avions « vécue» vraiment ${ }^{8}$. L'enjeu de cette " suspension volontaire de l'incrédulité » n'est pas de nous « leurrer », mais, tout au contraire, d'affiner notre connaissance de la réalité, d'élargir les frontières de notre expérience au-delà de notre vécu personnel ${ }^{9}$ en tirant profit d'une expérience imaginaire qui, pour avoir été inventée de toutes pièces, n'en est pas moins, sur bien des aspects, analogue à notre expérience vécue. Une condition est cependant requise pour que l'on ait envie de « feindre de croire » à l'histoire qui nous est racontée : l'histoire en question doit avoir l'apparence de la réalité, elle doit avoir l'apparence des choses que nous tenons pour " réelles », elle doit donc être vraisemblable. Et c'est pour cette raison que la notion d'imitation est, depuis l'Antiquité grecque, le pivot autour duquel gravite toute définition de la fiction. Exiger de la fiction qu'elle n'imite plus la réalité, exiger des romanciers qu'ils étoffent leurs récits de situations invraisemblables ou qu'ils se limitent à "réécrire » les œuvres déjà écrites ( on écrit des livres uniquement sur d'autres livres ou autour d'autres livres» Eco 1983: 522) revient à condamner la fiction à ne plus exister (ou à continuer à exister sous un forme qui n'est plus compatible avec la mission qu'elle a rempli dans notre tradition culturelle). Eco objecterait que le public actuel a désormais perdu la capacité de «feindre de croire » aux histoires divulguées par la fiction et qu'il finit donc par croire vraiment aux représentations irréelles qu'elle met en circulation. «Je crois qu'il n'y a plus de différence - fait-il dire à l'un de ses personnages - entre s'habituer à faire semblant de croire et s'habituer à croire " (Le Pendule de Foucault 1988 : 474). Soit. Mais pourquoi étendre le « donquichottisme » de certains lecteurs à l'ensemble de la société ? Pourquoi ne pas reconnaître qu'il s'agit d'un phénomène qui ne concerne que des franges minoritaires du public? Pourquoi Eco ne s'interroge-t-il jamais sur les causes historiques de cette vulnérabilité croissante d'une partie de l'imaginaire social à la fiction? Pourquoi accepte-t-il comme une fatalité ce dysfonctionnement de notre rapport à la fiction sans jamais le problématiser ? Pourquoi enfin, s'empresse-t-il, pour réhabiliter 
la fiction, d'injecter dans ses propres romans des doses massives d'invraisemblance, comme s'il fallait dissuader l'ensemble de ses lecteurs (à aucune exception près) de la tentation de tenir pour vraies les histoires qu'il raconte?

\section{La fiction selon Eco ou l'apothéose du nihilisme}

Le moment est venu de faire le bilan de ces regards croisés sur le phénomène du conspirationnisme. Selon le point de vue que j'ai essayé de défendre, la vulnérabilité de l'imaginaire social aux croyances véhiculées par le storytelling conspirationniste serait la conséquence du déficit de crédibilité dont souffrent, au sein de notre société, les valeurs éthiques, esthétiques, politiques et religieuses autour desquelles gravitait la vie symbolique des sociétés du passé. Le conspirationnisme serait donc, de mon point de vue, un symptôme du nihilisme croissant de notre société. Ce diagnostic concorde avec l'une des plus pénétrantes définitions du nihilisme que l'on puisse trouver sur le marché des idées philosophiques. Je pense, bien entendu, à ce célèbre chapitre du Crépuscule des idoles où Nietzsche entreprend d'expliquer «Comment le monde-vrai devint une fable ». Arrêtons-nous un instant sur cette assertion de Nietzsche et demandons-nous pourquoi la montée du nihilisme (c'est-à-dire, le désaveu, par notre société, des valeurs éthiques, politiques, esthétiques et religieuses) devrait avoir pour conséquence de nous condamner à vivre dans un monde colonisé par des scénarios fictionnels (selon un constat qui est partagé par Eco aussi, mais que celui-ci a le tort, de mon point de vue, d'accepter comme une fatalité sans vraiment s'interroger sur les causes historiques de cette « fictionnalisation du réel »). Il n'est possible, selon moi, de comprendre quelle connexion il peut y avoir entre le nihilisme et la "fictionnalisation du réel » (le monde-vrai qui devient une fable) qu'en admettant que les valeurs spirituelles autour desquelles gravite la vie sociale, loin d'être quelque chose de « séparé » de la réalité, se trouvent, bien au contraire, au cœur même des choses ou des faits que nous appelons " réalité ». Je ne suis pas en train de dire que les choses ont une essence spirituelle et que les formes sensibles à travers lesquelles nous les percevons ne sont que des apparences trompeuses. Mais il est évident qu'il n'y a aucune réalité que je puisse percevoir qui ne soit pas imprégnée de significations éthiques, politiques, esthétiques ou religieuses (ou qui ne pourrait l'être). Les réalités que nous percevons ne sont jamais, en effet, des réalités neutres : elles sont toujours connotées éthiquement, esthétiquement, politiquement ou religieusement (ou pourraient l'être). Nous ne nous limitons pas à faire le constat que certaines choses existent, nous les qualifions de bonnes ou de mauvaises, de belles ou de laides, de justes ou d'injustes, de sacrées ou de profanes. Ces caractérisations axiologiques ne sont pas quelque chose de superflu dont on pourrait très bien faire abstraction en laissant subsister uniquement les choses que nous percevons dans leur dimension ontologique brute. En plus d'avoir une existence (réelle ou imaginaire, empirique ou spirituelle), les choses ont aussi un sens et, justement à cause de ce sens, chacune d'entre elle retentit sur notre affectivité d'une manière spécifique, suivant la compatibilité ou l'incompatibilité de sa nature avec les valeurs éthiques, esthétiques, religieuses ou politiques en lesquelles nous croyons. Faire l'impasse sur cette dimension affective et axiologique signifie ne rien comprendre à la manière dont les êtres humains se rapportent à leur expérience vécue et lui donnent un sens. N'en déplaise à ceux qui voudraient porter sur l'expérience vécue de l'homme le même regard «scientifique » que les physiciens portent sur les protons et les neutrons ou les chimistes sur les particules élémentaires, il n'existe pas, à l'échelle 
humaine, une seule réalité qui ne soit pas connotée éthiquement, esthétiquement, politiquement ou religieusement (ou qui ne pourrait, le cas échéant, l'être). Que se passerait-il si l'on vidait la réalité de sa dimension axiologique en ne la faisant subsister que dans sa dimension ontologique primaire? La réalité deviendrait une matière molle que l'on pourrait former et déformer à souhait par toute sorte de manipulations (exactement comme le fait actuellement la technoscience sous l'impulsion de l'ultracapitalisme déréglé). Face à l'absence de tout frein d'ordre éthique, politique, esthétique ou religieux, rien ne nous interdirait, par exemple, d'indexer le réel sur l'irréel, le vraisemblable sur l'invraisemblable, le possible sur l'impossible. Dans un tel contexte, comment s'étonner si les scénarios invraisemblables colportés par le storytelling conspirationniste parviennent petit à petit à coloniser la vision sociale du réel ?

Mais la faute n'est pas à la fiction, si la société en est arrivée là. En tant que pratique mimétique, la fiction a même été, à travers l'histoire, l'un des remparts les plus efficaces contre le phénomène qui favorise la décomposition actuelle du sens de la réalité et la concomitante montée du crédo conspirationniste: le nihilisme. L'objet du mimétisme fictionnel n'est pas, en effet, la réalité matérielle, mais la réalité spirituelle: les auteurs de fiction n'imitent pas (ou n'imitent pas seulement) l'apparence extérieure de la réalité empirique, ils imitent aussi (et surtout) les significations éthiques, esthétiques, politiques et religieuses qui déterminent l'attitude affective de la société à l'égard de la réalité empirique. Ce faisant, ils ont ainsi pu s'acquitter d'une fonction culturelle majeure : la problématisation du rapport entre des situations tirées de l'expérience vécue et les catégories éthiques, esthétiques, politiques et religieuses qui, dans une conjoncture historique déterminée, fonctionnaient comme critère d'évaluation des situations imitées ${ }^{10}$. En peignant des situations inédites, mais vraisemblables, les artistes ont contribué à mettre le public en crise avec lui-même, ils l'ont obligé à questionner ses convictions éthiques, politiques, religieuses, à les reconsidérer à la lumière de circonstances peu ordinaires qui reproduisaient des comportements déviants (meurtre, adultère, inceste, ingratitude filiale, etc.) mais sans les présenter comme des errements, des aberrations ou des coups de folie. Madame Bovary est-elle une débauchée ou une victime? Raskolnikov est-il coupable ou innocent ? Julien Sorel est-il un simple voyou ou le pauvre représentant d'une génération postnapoléonienne égarée ? Flaubert, Dostoïevski, Stendhal ne le disent pas et, ce faisant, ils obligent chacun de leurs lecteurs à questionner ses convictions profondes en matière éthique, politique, religieuse ou esthétique.

Or, dans le cadre d'une société comme la nôtre, qui est de plus en plus régie par des mécanismes d'autorégulation économique, les idées qui modelaient autrefois les comportements humains non reconductibles à des intérêts économiques (les idées éthiques, politiques, religieuses, esthétiques, etc.) n'ont plus besoin d'être problématisées. Je dirais même qu'elles ne doivent plus l'être: toute problématisation ralentirait leur agonie fatale et ralentirait, surtout, la réalisation du programme utopique ${ }^{11}$ (et inhumain) qui tient actuellement en otage le destin de l'humanité. Le modèle d'organisation sociale en question est un modèle de gestion exclusivement économique du social. En le qualifiant d'« exclusivement économique », j'entends dire que tout recours à des instruments de cohésion sociale qui ne soient pas de nature économique y est catégoriquement « exclu ». La cohésion sociale est désormais assurée par le bien-être matériel, succédané de toutes les valeurs éthiques, politiques, esthétiques et religieuses qui, autrefois, dans des sociétés moins matérialistes que la nôtre, servaient à réconcilier les hommes avec la vie. Pour accélérer l'avènement d'une société entièrement gouvernée par des réflexes, des instincts 
et des pulsions de nature économique, l'ultra-capitalisme actuel ne peut pas admettre qu'il puisse y avoir des pratiques symboliques capables de stimuler l'imaginaire collectif par la mimésis de situations réelles qui questionnent, problématisent ou mettent en crise les évidences éthiques, politiques ou religieuses dominantes. Il faudra alors dénoncer la dangerosité des conventions culturelles (le "pacte fictionnel») sur lesquelles se fonde l'interaction de la société avec les genres imaginaires et accréditer l'idée baroque et saugrenue selon laquelle la fiction serait la cause des mystérieuses contaminations psychotiques qui polluent actuellement le sens de la réalité.

Le diagnostic d'Eco sur les théories du complot n'est pas à refuser uniquement parce qu'il est erroné (ou partiellement erroné). Il est à refuser, selon moi, parce qu'il fait le jeu du capitalisme, en secondant la logique qui est en train de renfermer la vie humaine à l'intérieur d'un horizon exclusivement économique. Le modèle de fiction dont relèvent ses romans emprisonne les représentations fictionnelles dans une sous-sphère ontologique, correspondant à tout ce à quoi nous ne pouvons pas croire à cause de son invraisemblance. Miroir du faux, la fiction devient, chez lui, une activité ludique et oiseuse, n'ayant plus aucun rapport avec la réalité et ne répondant, surtout, à aucune autre finalité qu'à celle de satisfaire cette sorte d'onanisme érudit dans lequel se complaisent les adeptes postmodernes de l'intertextualité, de la métatextualité, de la paratextualité, de l'hypertextualité et, plus en général, de l'écriture au second degré. On dit qu'un écrivain devient célèbre quand il sait interpréter le zeigeist, l'esprit de son temps. Le problème d'Umberto Eco est qu'il n'a pas interprété le zeitgeist, il l'a incarné, il l'a épousé, il en est devenu le chantre officiel.

\section{BIBLIOGRAPHY}

Eco, U. (2000), Baudolino. Paris : Grasset.

Eco, U. (1988), Le Pendule de Foucault. Paris : Grasset.

Eco, U. (2010), Le Cimetière de Prague. Paris : Grasset.

Eco, U. (2015), Numéro zéro. Paris : Grasset.

Eco, U. (1996), L'T̂le du jour d'avant. Paris : Grasset.

Eco, U. (1996), Six promenades dans les bois du roman et d'ailleurs. Paris : Grasset.

Eco, U. (1979), Lector in fabula. Milano : Bompiani.

Eco, U. (1983), « Postille a Il nome della rosa » dans Il nome della rosa. Milano : Bompiani.

Foucault, M. (1976), Histoire de la sexualité I. Paris : Gallimard.

Fukuyama, F. (1992), La fin de l'histoire et le dernier homme. Paris : Flammarion.

Herman, D., Jahn, M., Ryan, M.-L. (eds.) (2004), Routledge Encyclopedia of Narrative Theory, London New York : Routledge.

Hume, D. (1995 [1735-1737]), Traité de la nature humaine. Paris : Flammarion. 
Michéa, J.-C. (2007), L'empire du moindre mal. Essai sur la civilisation libérale. Paris : Climats.

Preve, C. (2007), Storia dell'etica. Pistoia : Petite plaisance.

Rosanvallon, P. (1979), Le capitalisme utopique. Histoire de l'idée de marché. Paris : Seuil.

Schaeffer, J.-M. (1999), Pourquoi la fiction? Paris : Seuil.

Vattimo, G. (1985), La fine della modernità. Milano : Garzanti.

Weber, M. (2003 [1922]), Economie et société, chapitre 1, Paris : Plon.

\section{NOTES}

1. Parfois, cette tâche est confiée à un personnage agissant dans l'univers diégétique : "Tu sei felice di essere stato ancora una volta il Principe della Menzogna, e te ne glori » dit Niceta à Baudolino.

2. Ce jugement est répété plusieurs fois par Aristote. Voir, par exemple, Poétique, 25, 1461 b.

3. La lettre du père Rodin au père Roothaan dans les Mystères du peuple de Sue et le modèle du pamphlet de Maurice Joly, Dialogue aux enfers entre Machiavel et Montesquieu ou la politique de Machiavel au XIX siècle, par un contemporain, dialogue qui servira, à son tour, de modèle aux Protocoles des Sages de Sion. Eco a retracé la généalogie romanesque des croyances conspirationnistes dans les Promenades dans les bois du roman et ailleurs. (Eco : 1996).

4. Cf. L'ouverture du roman Joseph Balsamo.

5. Rabbi Löw est l'un des plus grands rabbins des temps modernes, auteur de la légende du Golem, monstre dont Dieu se servirait pour venger les persécutions subies par les Juifs. Des traces de cette légende se trouvent également dans les contes des frères Grimm et dans Frankenstein de Mary Shelley.

6. Il convient d'ajouter aux auteurs déjà cités (Sue et Dumas), la liste des écrivains suivants, dont Eco a montré, dans le Cimetière de Prague, qu'ils ont contribué à enraciner dans l'imaginaire social des croyances antisémites par l'intermédiaire de livres de fiction: Alphonse Toussenel, Lord Beaconsfield, alias Disraeli, Jakob Brafmann, Gougenot des Mousseaux, Marie Joseph Gabriel Antoine Jogand-Pagès dit Léo Taxil.

7. Pour feindre de croire à ce qu'un auteur de fiction me raconte, il faut que les significations qu'il exprime aient l'apparence des choses auxquelles, généralement, je crois : la réalité. Nous appelons, en effet, "réelles" les choses auxquelles nous croyons, les choses que nous considérons comme dignes de confiance, la croyance n'étant rien d'autre, à bien y réfléchir, que le sentiment que nous ressentons face aux entités avec lesquelles nous avons la plus grande familiarité (David Hume 1995 [1735-1737]). Les comportements humains représentés dans un roman, dans un film ou dans une pièce de théâtre ne peuvent donc être considérés comme une «fiction» que parce qu'ils "font semblant» d'être des comportements "réels ». Il doit alors forcément y avoir une quelconque correspondance entre les comportements en question et les formes à travers lesquelles la société tend à se représenter l'agir humain. C'est pour cette raison que les auteurs de fictions déploient leur art sur le mode de l'imitation.

8. «... lorsque je lis La vie de Marianne, je n'ai accès à l'univers fictionnel que pour autant que je réactive la feintise ludique de Marivaux, c'est-à-dire pour autant que j'adopte la posture qui serait la mienne si une personne nommée Marianne me racontait sa vie, à moins que je ne m'identifie fictivement à Marianne elle-même racontant son histoire " J.-M. Schaeffer 1999 : 228.

9. «... narrative, in its fictional form, widens our mental universe beyond the actual and the familiar and provides a playfield for thought experiments " Herman, D., Jahn, M., Ryan, M.-L. (eds.) $2004: 345$. 
10. Du fait que l'homme est un animal politique et qu'il doit s'associer avec des personnes qui partagent ses valeurs, il ne s'ensuit pas qu'il soit un animal grégaire : il peut refuser les valeurs auxquels ses semblables ont fait allégeance, s'il estime qu'elles sont contraires à ses convictions. Mais il a besoin pour cela de pratiques culturelles qui tiennent en exercice sa capacité de critiquer les évidences autour desquelles les croyances sociales se sont coagulées. Or, dans notre tradition culturelle, la fiction a, en partie, rempli cette fonction.

11. Cf. Pierre Rosanvallon 1979.

\section{ABSTRACTS}

Selon U. Eco, l'inflation des théories du complot, des fausses nouvelles et d'autres croyances aberrantes de ce type relèverait de l'influence néfaste de la fiction sur l'imaginaire social. Dès lors, les règles qui président au rapport que la société entretient avec les genres fictionnels (le "pacte fictionnel») devraient être sérieusement réformées afin de préserver les certitudes résiduelles qui permettent à notre société de garder encore intacte sa culture et son unité. Le présent article critique les prises de position d'Eco contre la fiction en montrant que celle-ci est, dans notre tradition culturelle, l'un des lieux principaux de problématisation des croyances éthiques, esthétiques, politiques et religieuses. Loin de corrompre la perception sociale de la réalité, la fiction peut servir à mettre à l'épreuve les normes d'ordre éthique, politique, esthétique et religieux qui régissent la vie sociale.

Secondo U. Eco, l'inflazione delle teorie del complotto, delle false notizie e di altre credenze aberranti di questo tipo dipenderebbe dall'influenza nefasta che la finzione esercita sull'immaginario sociale. Le regole che presiedono al rapporto tra la società e i generi immaginari (il cosiddetto "patto finzionale") dovrebbero allora essere seriamente ripensate al fine di preservare le residue certezze che permettono alla nostra società di conservare ancora intatta la propria cultura e la propria coesione. Il presente lavoro critica le prese di posizione di Eco contro la finzione mostrando che quest'ultima è, nella nostra tradizione culturale, uno dei luoghi principali di problematizzazione delle credenze etiche, estetiche, politiche e religiose. Lungi dal corrompere la percezione sociale della realtà, la finzione può servire a mettere alla prova le norme di carattere etico, politico, estetico e religioso che reggono la vita sociale.

\section{INDEX}

Mots-clés: fiction, Eco Umberto, argumentation, storytelling, théorie du complot

\section{AUTHOR}

\section{ALESSANDRO LEIDUAN}

Université de Toulon. Babel, 2649 